\title{
Ultrahigh Thermoelectric Performance in Mosaic Crystals
}

\author{
Ying He, Ping Lu, Xun Shi, * Fangfang Xu, * Tiansong Zhang, Gerald Jeffrey Snyder, \\ Ctirad Uher, and Lidong Chen*
}

Thermoelectric technology has the advantages of no moving components, no noise signal, minimal environmental pollution and, above all, exceptional service reliability and good portability. ${ }^{[1,2]}$ The energy conversion efficiency is generally evaluated by the dimensionless thermoelectric figure of merit $z T\left(z T=S^{2} \sigma T / \kappa\right)$, where $S$ is the Seebeck coefficient, often called the thermopower, $\sigma$ is the electrical conductivity, $T$ is the absolute temperature, and $\kappa$ is the thermal conductivity which includes both the electronic contribution $\kappa_{e}$ and the lattice thermal conductivity $\kappa_{L} \cdot{ }^{[1-4]}$ Excellent thermoelectric performance requires highly efficient electronic transport combined with a very poor ability to conduct heat. The above two requirements are substantially orthogonal and difficult to realize in practice and are the primary reason why thermoelectricity has had so far a rather limited commercial use. The interdependence of transport parameters prevents a simultaneous optimization of both electronic and thermal transport properties and, instead, the design of an effective thermoelectric material is limited to tuning either the electronic transport ${ }^{[2,5,6]}$ or to minimizing the lattice thermal conductivity. ${ }^{[2,7-14]}$

In crystalline materials, the grain size covers the range from a few nanometers to the physical extent of the sample which can be several centimeters. A single crystal is an extreme case

Dr. Y. He, Dr. P. Lu, Prof. X. Shi, Prof. F. Xu, Prof. L. Chen State Key Laboratory of High Performance

Ceramics and Superfine Microstructure

Shanghai Institute of Ceramics

Chinese Academy of Sciences

Shanghai 200050, China

E-mail:xshi@mail.sic.ac.cn; ffxu@mail.sic.ac.cn; cld@mail.sic.ac.cn

Dr. Y. He, Prof. X. Shi, T. Zhang, Prof. L. Chen

CAS Key Laboratory of Materials for Energy Conversion

Shanghai Institute of Ceramics

Chinese Academy of Sciences

Shanghai 200050, China

Dr. Y. He, Dr. P. Lu

University of Chinese Academy of Sciences

Beijing 100049, China

Prof. G. J. Snyder

Department of Materials Science and Engineering

Northwestern University

Evanston IL, 60208 USA

Prof. C. Uher

Department of Physics

University of Michigan

Ann Arbor, MI 48109, USA

Prof. L. Chen

Shanghai Institute of Materials

Genome, Shanghai 200444, China

DOI: 10.1002/adma.201501030 of a crystalline material where the translational symmetry of the lattice extends to the edges of the sample. If a material is composed of many crystallites of various sizes and orientation, see Figure 1a, it is referred to as a polycrystalline specimen. As the grain size becomes smaller and smaller and reaches a few nanometers or less, the material is generally refered to as a nanocrystalline solid. The vast spectrum of crystallite sizes spanning some five orders of magnitude has a dramatic effect on the charge and phonon transport and presents an opportunity to tune the transport behavior of solids and, consequently, their thermoelectric performance. Due to the absence of boundary or interface scattering, a single crystal possesses both excellent electrical and thermal conductivities, the former being an advantage for thermoelectricity while the

(a)

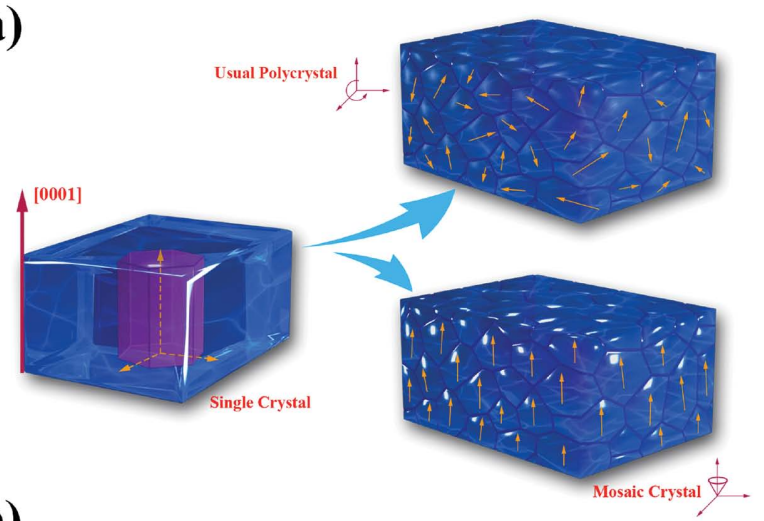

(b)

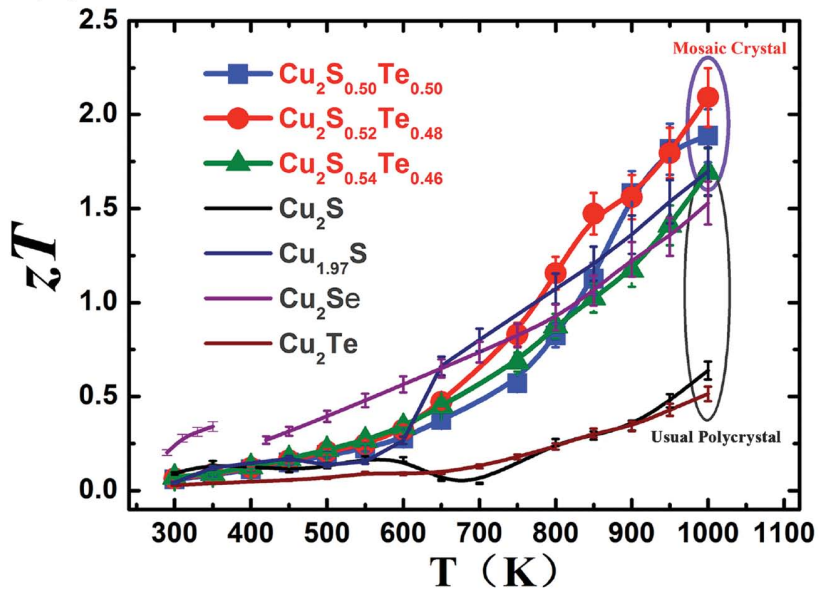

Figure 1. Enhanced $z T$ values in mosaic crystals. a) Structural characteristics of a single crystal, a usual polycrystal, and a mosaic crystal. Arrows refer to alignment of crystal grains. b) Temperature dependence of $z T$ values for $\mathrm{Cu}_{2}(\mathrm{~S}, \mathrm{Te})$ mosaic crystals. The measurement uncertainty of $z T$ is about $15 \%$. 


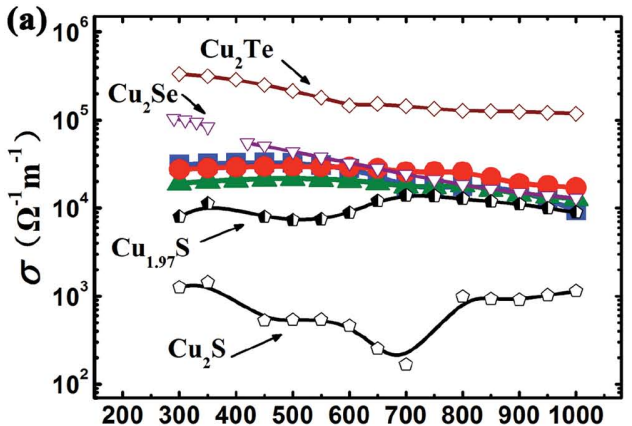

(c)

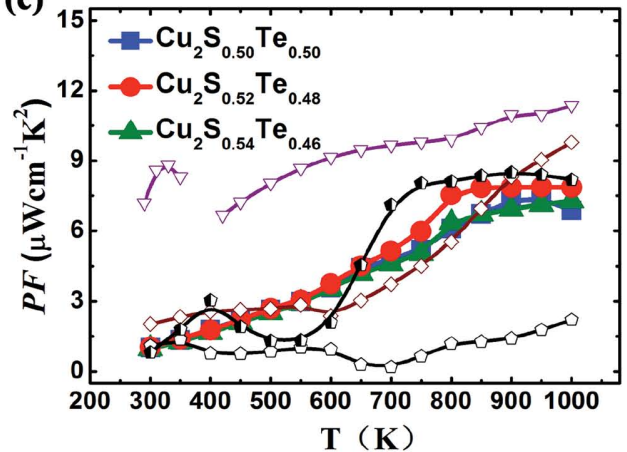

(b)

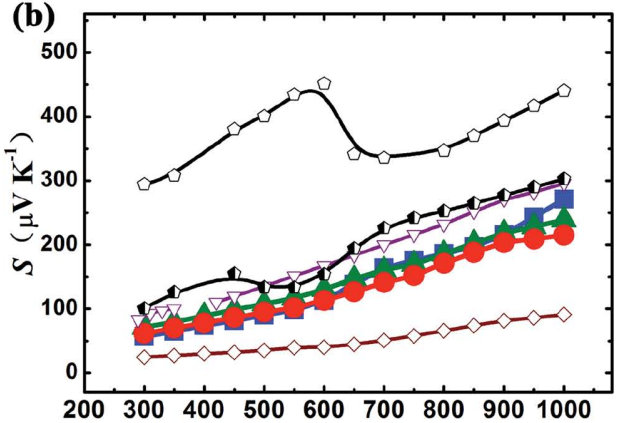

(d)

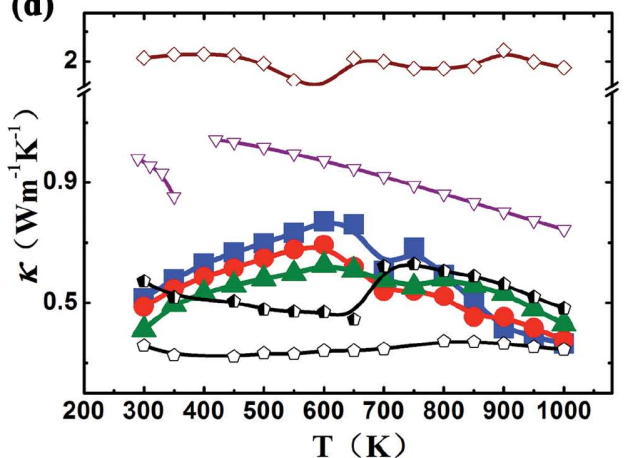

Figure 2. Temperature dependence of thermoelectric properties in $\mathrm{Cu}_{2}(\mathrm{~S}, \mathrm{Te})$ mosaic crystals. a) Electrical conductivity $(\sigma)$. b) Seebeck coefficient $(S)$. c) Power factor (PF). d) Thermal conductivity $(\kappa)$.

latter is not so. In contrast, due to extremely effective scattering on boundaries and interfaces, a nanomaterial usually has both low electron and phonon conduction. Optimization of thermoelectric properties making use of the above two extreme crystalization regimes has been shown very successful in many families of thermoelectric materials in the past decades, ${ }^{[3-14]}$ and the $z$ Ts above 2 in bulk materials are reported for these two extremes, i.e., perfect single crystals in $\mathrm{SnSe}^{[8]}$ and PbTe-based nanocomposites. ${ }^{[7]}$

In this work, we go beyond the traditional approaches that use single crystals $^{[8]}$ or conventional nanomaterials, ${ }^{[3,4,7,9-12]}$ i.e., which rely on either enhancing or degrading electron and phonon conduction synchronously. Instead, by using the concept of nanoscale mosaicity in a single-phase polycrystalline material, we achieve a simultaneous enhancement of several thermoelectric parameters resulting in a record-high $z T$ value of $\approx 2.1$ at $1000 \mathrm{~K}$ in $\mathrm{Pb}$-free bulk nanomaterials. A mosaic crystal is a very special state proposed by Darwin, ${ }^{[15]}$ involving high degree of perfection in the lattice translations throughout the crystal. The crystal is composed of mosaic blocks with each block being a perfect crystal but titled or rotated with respect to others by a fraction of a minute of arc. Different from the random orientations in ordinary polycrystals or nanocrystal materials, blocks in a mosaic crystal exhibit a nearly identical orientation thereby the bulk appears like a single crystal from the macroscale point of view but contains a number of smallangle boundaries ${ }^{[15-18]}$ (see Figure 1a). High lattice coherence in a mosaic crystal is expected to result in excellent electronic transport just as in a single crystal while a minute misorientation of grain boundaries is still very effective in scattering heat conducting phonons, leading to exceptional thermoelectric properties. In the present study, we achieved the desired mosaic structure in $\mathrm{Cu}_{2}(\mathrm{~S}, \mathrm{Te})$ bulk single-phase polycrystalline materials where microscale quasi-single crystals consist of 10-20 nm mosaic grains. Compared with the usual polycrystalline matrix samples such as $\mathrm{Cu}_{2} \mathrm{~S}$ and $\mathrm{Cu}_{2} \mathrm{Te}$, the $z T s$ are increased by about a factor of three due to the costrengthened thermoelectric parameters (Figure 1b). This mosaic-based strategy can be applied with other successful approaches to further improve the performance of thermoelectric materials.

We use the traditional solid-state reaction as well as the spark plasma sintering method to obtain bulk nanomaterials. Although Te has a much larger atomic radius $(1.70 \AA)$ than S (1.04 A), surprisingly, we obtain complete solid solutions between $\mathrm{Cu}_{2} \mathrm{~S}$ and $\mathrm{Cu}_{2} \mathrm{Te}$ (see XRD in Figure S1, Supporting Information). However, different from the $\mathrm{Cu}_{2} \mathrm{X}(\mathrm{X}=\mathrm{S}, \mathrm{Te})$ matrix which undergoes at least three structural transformations above room temperature, ${ }^{[13,19,20]} \mathrm{Cu}_{2}(\mathrm{~S}, \mathrm{Te})$ attains only two structural forms (see Figure S2, Supporting Information) and the phase transition tempearture is determined by the $\mathrm{S} /$ Te atomic ratio. We have chosen chemical compositions around $\mathrm{Cu}_{2} \mathrm{~S}_{0.5} \mathrm{Te}_{0.5}$ because the latter composition has a strong mosaic character (see below). It crystallizes in a hexagonal structure at room temperature, similar to the intermediate temperature phases of $\mathrm{Cu}_{2} \mathrm{~S}$ and $\mathrm{Cu}_{2} \mathrm{Te}$.

The temperature dependence of thermoelectric transport parameters between 300 and $1000 \mathrm{~K}$ is shown in Figure 2. Due to the natural deficiency of copper, all samples show p-type conducting behavior. The electrical conductivity is on the order of $10^{4} \Omega^{-1} \mathrm{~m}^{-1}$ in the whole temperature range, lying between the conductivities of $\mathrm{Cu}_{2} \mathrm{~S}$ and $\mathrm{Cu}_{2} \mathrm{Te}$. The room temperature thermopower is around $60 \mu \mathrm{V} \mathrm{K} \mathrm{K}^{-1}$, again in between the values of $\mathrm{Cu}_{2} \mathrm{~S}\left(\approx 300 \mu \mathrm{V} \mathrm{K}^{-1}\right)$ and $\mathrm{Cu}_{2} \mathrm{Te}\left(\approx 25 \mu \mathrm{V} \mathrm{K}^{-1}\right)$. The thermopower increases monotonously to about $260 \mu \mathrm{V} \mathrm{K}^{-1}$ at $1000 \mathrm{~K}$ and no 


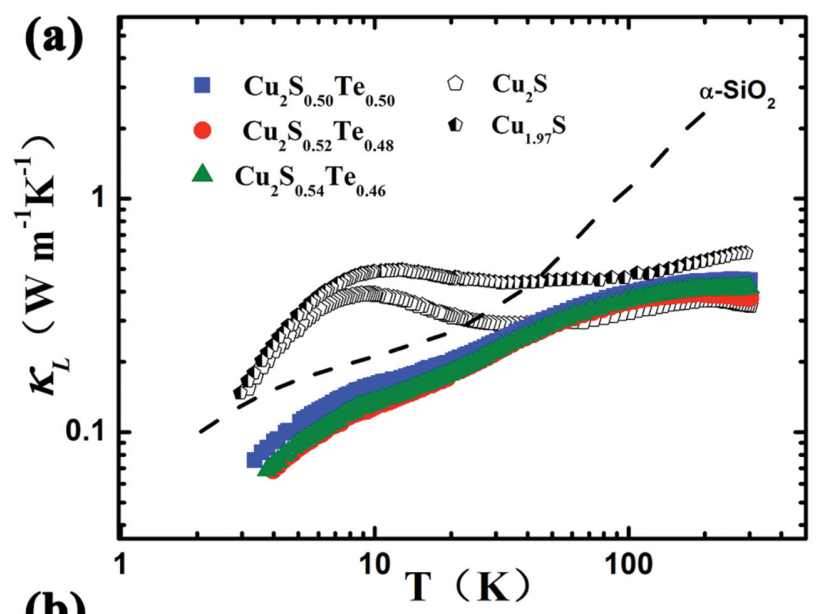

(b)

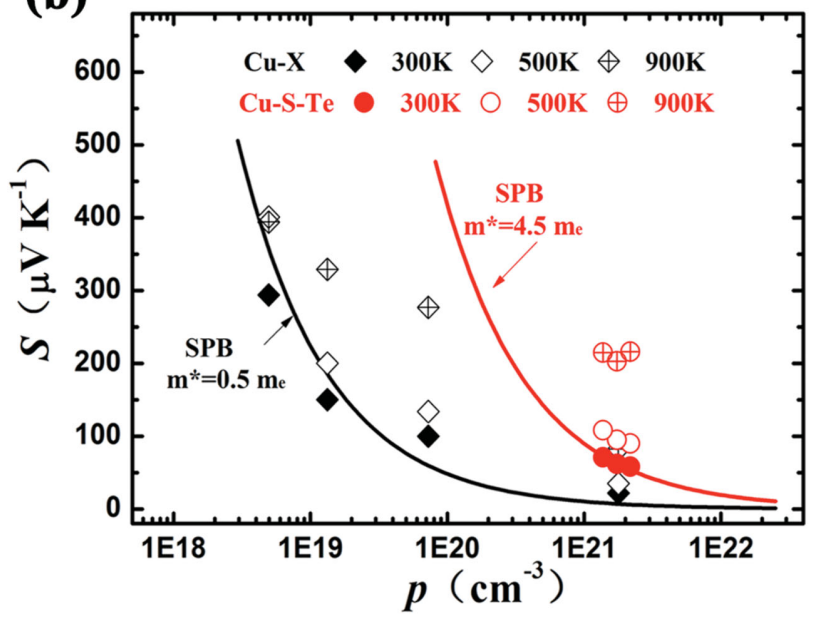

Figure 3. Abnormal electrical and thermal transports in $\mathrm{Cu}_{2}(\mathrm{~S}, \mathrm{Te})$ mosaic crystals. a) Low temperature lattice thermal conductivity. The data for $\mathrm{Cu}_{2} \mathrm{Te}$ is not shown because the electronic contribution dominates the total thermal conductivity, leading to huge error bars for the estimated lattice thermal conductivity. b) Relationship between the Seebeck coefficient and the carrier concentration at various temperatures.

peaks are observed, suggesting that the band gap of our samples should be similar to $\mathrm{Cu}_{2} \mathrm{~S}(1.2 \mathrm{eV})$ or $\mathrm{Cu}_{2} \mathrm{Te}(1.04 \mathrm{eV})$.

The solid solution samples possess an extremely low thermal conductivity with the largest total values not exceeding $0.8 \mathrm{~W} \mathrm{~m}^{-1} \mathrm{~K}^{-1}$ at $650 \mathrm{~K}$ and $0.5 \mathrm{~W} \mathrm{~m}^{-1} \mathrm{~K}^{-1}$ at $1000 \mathrm{~K}$. Such values are actually lower than the lattice thermal conductivity contribution in many state-of-the-art thermoelectric materials. The electronic thermal conductivity is calculated by $\kappa_{e}=L_{0} \sigma T$, where $L_{0}$ is the Lorenz number with an estimated value of $1.6 \times 10^{-8} \mathrm{~V}^{2} \mathrm{~K}^{-2}$ at high temperatures based on the single band model and electron-phonon interactions. The $\kappa_{\mathrm{L}}$ is then calculated by subtracting the $\kappa_{\mathrm{e}}$ from the total $\kappa$, which is shown Figure S3, Supporting Information. In the temperature range from 2 to $1000 \mathrm{~K}$, the $\kappa_{\mathrm{L}}$ falls below $0.3-0.4 \mathrm{~W} \mathrm{~m}^{-1} \mathrm{~K}^{-1}$, a very low value for a fully densified solid. More interestingly, the solid solutions are similar to cage-clathrates ${ }^{[21]}$ to display a glass-like behavior at low temperatures typified by plateaus observed between 10 and $20 \mathrm{~K}$ (see Figure 3a). This behavior stands in a stark contrast with the temperature dependence of thermal conductivity of $\mathrm{Cu}_{2} \mathrm{~S}$ which exhibits a distinctly crystalline character with the pronounced dielectric peak near $10 \mathrm{~K}$.

Apart from the exceptionally low thermal conductivity, the solid solutions also show extremely abnormal electronic transport. Figure $3 \mathrm{~b}$ shows the thermopower as a function of carrier concentration at various temperatures. Solid solution samples clearly show much higher thermopowers than the Pisarenko relation based on the $\mathrm{Cu}_{2} \mathrm{~S}$ and $\mathrm{Cu}_{2}$ Te data from 300 to $1000 \mathrm{~K}$. In particular, solid solutions display similar carrier concentrations to those in $\mathrm{Cu}_{2} \mathrm{Te}$ (see Figure $\mathrm{S} 4$, Supporting Information), but their thermopowers are at least twice as large. This is very unusual because enhancements of such magnitude in the thermopower (for comparable carrier densities) have only been reported for cases where resonant states were believed to be effective (Tl-doped $\mathrm{PbTe}{ }^{[5]}$ ), when the thermopower was governed by critical electrons during second-order phase transitions in $\mathrm{Cu}_{2} \mathrm{Se},{ }^{[22,23]}$ and in the presence of unbalanced spins and orbital degrees of freedom among magnetic ions with different charge states in cobalt oxides. ${ }^{[24]}$ Using the measured thermopower and carrier concentrations, the electron effective masses were calculated based on the single parabolic band model. The results show a nearly one order of magnitude enhancement at room temperature, an extremely unexpected value in the context of the usual solid solution materials.

The well-tuned electronic transport properties bode well for a competitive thermoelectric power factor $P F$ defined as $P F=\sigma S^{2}$. In fact, the solid solution samples have similar or slightly lower power factors $\left(8 \mu \mathrm{W} \mathrm{cm}{ }^{-1} \mathrm{~K}^{-2}\right.$ at $\left.1000 \mathrm{~K}\right)$ compared to $\mathrm{Cu}_{2} \mathrm{Te}$, $\mathrm{Cu}_{2} \mathrm{Se}$, or $\mathrm{Cu}_{1.97} \mathrm{~S}$ (Figure 2), ${ }^{[13,14]}$ the best thermoelectric materials among the binary $\mathrm{Cu}_{2-\delta} \mathrm{X}$ compounds, but also have much lower thermal conductivity. Overall, the three critical transport parameters of copper-based solid solution samples-electrical conductivity, thermopower, and thermal conductivity-combine to yield ultrahigh $z T s$ values near or above 2. Specifically, a maximum $z T$ of 1.7 for $\mathrm{Cu}_{2} \mathrm{~S}_{0.54} \mathrm{Te}_{0.46}, 1.9$ for $\mathrm{Cu}_{2} \mathrm{~S}_{0.50} \mathrm{Te}_{0.50}$, and 2.1 for $\mathrm{Cu}_{2} \mathrm{~S}_{0.52} \mathrm{Te}_{0.48}$ are achieved near $1000 \mathrm{~K}$. The values are significantly higher than the values measured on conventional polycrystalline materials (Figure 1b).

Why the solid solutions possess instantaneously good electronic transport and glass-like exceptionally low thermal conductivity must be related to the unusual micro/nano structure of the material. We carried out the transmission electron microscopy (TEM) analyses using ultrathin sectioned samples (see Figure 4 and the Supporting Information) to gain insight into structural properties of solid solutions. Ignoring the mesh-like image from the perforated supporting carbon foil, Figure 4a shows uniform contrast throughout the area with the linear dimension of some $10 \mu \mathrm{m}$. Selected-area electron diffraction from anywhere in this imaged region illustrates a nearperfect single crystal-type [0001] zone-axis pattern (an example is shown in Figure $4 \mathrm{~b}$ acquired from the area marked by a red circle in Figure 4a) though short bars rather than dots in the reflection pattern suggest the presence of tiny degree of misorientation. Hitherto, the sectioned sample appears to be a single crystal with a minute amount of imperfections. However, both bright-field and dark-field images at amplified magnifications reveal an unexpected "polycrystalline-type" microstructure consisting of nanocrystallites of around $10-20 \mathrm{~nm}$ in size. This 

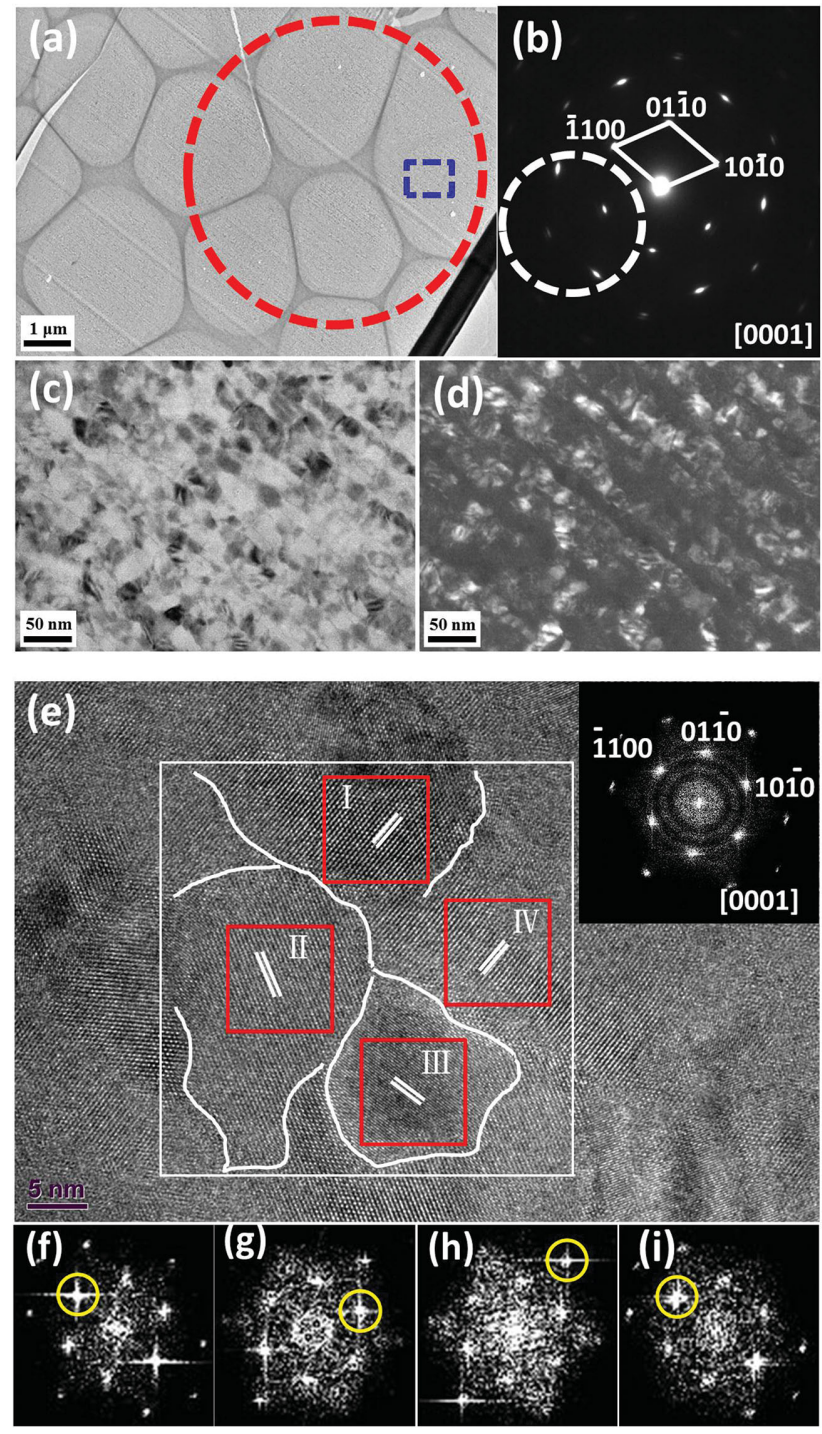

Figure 4. TEM and HRTEM analyses of the mosaic crystal $\mathrm{Cu}_{2} \mathrm{~S}_{0.5} \mathrm{Te}_{0.5}$. a) Bright-field image of the ultra-thin sectioned sample. b) Electron diffraction pattern of a large area (circumvented by a red circle in (a)) indicating a 'quasi-single crystalline' structure. c,d) Bright-field and dark-field images (using four diffraction spots marked with a white circle in (b)) of the framed area (blue frame in (a)), respectively. e) HRTEM image and its FFT diffractogram. $\mathrm{f}-\mathrm{i}$ ) FFT diffractograms of selected areas in (e) labeled as I, II, III, and IV, respectively. The nanocrystallites show nearly identical diffractograms except for variations in the brightness of different reflections that correspond to a slight misorientation with respect to each other (bright reflections are highlighted by yellow circles in $(f-i)$ ).

mosaic feature is also observed and confirmed in the powder samples (see Figure S9, Supporting Information).

Chemical micro-analysis using the energy dispersive spectrometry (EDS) in TEM indicates a basically homogenous distribution of $\mathrm{S}$ and Te elements, further confirming the uniform nature of solid solutions (see Figure S5, Supporting Information). These analytical results indicate that we have obtained a typical architecture characteristic of the mosaic crystal, i.e., quasi-single crystals composed of nanocrystallites having the same phase structure, close chemical compositions, and nearly identical orientation. In order to examine the degree of misorientation among the nanocrystallites, high-resolution transmission electron microscopy (HRTEM) analysis was performed as shown in Figure 4e-i. The fast Fourier transform (FFT) of the diffractogram (top right corner) from a region framed by the white square in Figure 4e again shows a single crystal-type zone-axis pattern although it contains at least four nanocrystallites (outlined in white) of 15-20 nm. These four nanograins exhibit identical diffractograms (see Figure $4 \mathrm{f}-\mathrm{i}$, respectively) except for some variation in the brightness of reflections. The intensity of reflections is proportional to the degree of favoring the Bragg condition for certain crystalline planes. Different distribution of reflection intensities (brightest reflections are highlighted using yellow circles in each diffractogram) suggests a slight misorientation in zone axes among these grains. The degree of misorientation is estimated as being within tens of milliradians.

The above structural analysis demonstrates that we have obtained a typical nanoscale mosaic microstructure in polycrystalline grains. This unique microstructure is quite different from the conventional nanocrystals or nanocomposite ${ }^{[3,4,7,9-12]}$ materials in which nanograins are oriented completely randomly and their misaligned interfaces would strongly affect electron transfer. In mosaic crystals, the very small mosaic grains or blocks suggest an ideal example of a lower dimensional structure imported into bulk materials which promotes excellent thermoelectric performance and in which strong quantum confinement effects and perhaps even electron barrier filtering result in much enhanced electron effective masses and thus thermopowers (see Figure 3b). ${ }^{[3,4,11]}$ Mosaic crystals contain a number of small angle tilt or rotate boundaries that will introduce long range disorder for thermal phonons, hence they acquire the glass-like, low thermal conductivity (see Figure 3a). Thus the unusual mosaicity composed of nanocrystals with tiny misorientations provides naturally independent pathways for electrons and phonons, i.e., the electrons transfer along the nearly coherent crystalline framework, while the phonons are frequently blocked by the strained lattices due to a dense network of small-angle tilted or rotated boundaries. The former makes the material behave like a crystal from the perspective of electrons while the latter makes it function as a glassy material as far as phonons are concerned. Consequently, nanoscale mosaicity can truly achieve the character of "phonon-glass electron-crystal," a classic thermoelectric concept proposed by Slack. ${ }^{[25]}$

In general, to form a mosaic crystal, flash-cooling is used during which structural defects do not have enough time to diffuse the surfaces while they have to accumulate to form boundaries. ${ }^{[15-18]}$ In the meantime, possible compositional variation develops, resulting in a large internal stress to further underscore the imperfection of the crystals. However, in the present study, we use the traditional long-term annealing procedure to prepare solid solution materials. Due to the extremely large difference in atomic sizes of $\mathrm{S}$ and $\mathrm{Te}$ (about 70\% mismatch), the solubility of $\mathrm{S}$ in $\mathrm{Cu}_{2} \mathrm{Te}$ or $\mathrm{Te}$ in $\mathrm{Cu}_{2} \mathrm{~S}$ is normally considered very small because the size tolerance for a solid solution is usually less than $15 \%$. However, here it provides another possibility to form mosaic crystals in which the long-range electron interactions are expected to lower the system's energy. The 
large internal stress arising from the very large atomic size mismatch can only be tolerated within a very small sample volume above which structural defects including subgrain boundaries must be formed to relax the lattice strains. Even a slight variation in the $\mathrm{S} / \mathrm{Te}$ atomic ratio may cause a large lattice strain, further promoting nanocrystallization. The coulombic interactions between cations and anions can also contribute to stabilize the structure, but the full understanding of the mechanism requires further study.

We also tested the thermal stability of the microstructure of our mosaic crystals by in situ TEM (see Figures S6 and S7, Supporting Information). Upon heating to nearly $500 \mathrm{~K}$, the nanograins start to coarsen similar to an Ostwald ripening process. ${ }^{[26]}$ During the whole heating process up to $973 \mathrm{~K}$, the electron diffraction maintains the single crystal-type zone axis pattern. Thus, the mosaic architecture is thermally presented in the solid solution materials up to near $1000 \mathrm{~K}$.

The strategy of nanoscale mosaicity goes beyond the previous approaches by providing multiform effects to tune thermoelectric properties. In this way, electrons are freely transferred along the frames of quasi-single crystals while phonons are strongly scattered by lattice strains or interfaces of mosaic nanograins. The optimization of the electron and phonon transport is simultaneously promoted to achieve ultrahigh thermoelectric performance. A $z T$ value of 2.1 is reported in the present Cu-based bulk nanomaterials. This strategy is expected to be applicable to any bulk thermoelectric materials by using a flash cooling technique or a mixture of elements with vastly mismatched atomic sizes to produce mosaic nanocrystals. It is also believed that this strategy can be easily applied together with the current successful approaches such as band structure engineering to further enhance thermoelectric performance.

\section{Experimental Section}

Materials and Methods: Pure element Cu (shots, 99.999\%, Alfa Aesar) S (pieces, 99.999\%, Alfa Aesar), and Te (pieces, 99.999\%, Alfa Aesar). All samples were prepared using a traditional solid state method. Starting materials were weighted in the ratio of the chemical formula, and then were sealed in silica tubes in a glove box under an $\mathrm{Ar}$ atomsphere (oxygen pressure $<0.1 \mathrm{ppm}$, water pressure $<0.1 \mathrm{ppm}$ ). The mixed elements were heated at the speed of $4 \mathrm{~K} \mathrm{~min}^{-1}$ to $1383 \mathrm{~K}$ and then maintained at this temperature for $10 \mathrm{~h}$ to ensure complete melting. Subsequently, the silica tubes were quenched in ice water. The obtained ingots were ground into powders and the powders were pressed into pellets. Next, the pellets were sealed in silica tubes in a glove box again and were annealed at $833 \mathrm{~K}$ for $7 \mathrm{~d}$. Finally, the obtained pellets were crushed into fine powders and consolidated by spark plasma sintering (Sumitomo SPS-2040) using a graphite mould with the diameter of $13 \mathrm{~mm}$ at around $713-723 \mathrm{~K}$ under a pressure of $65 \mathrm{MPa}$ for $5 \mathrm{~min}$. All the obtained solid solution samples are polycrystalline materials with wide range of grain size and random orientation (see Figure S8, Supporting Information) and their transports are expected isotropic. Thus, the bulk polycrystalline materials are used for the thermoelectric property measurement.

High-temperature Seebeck coefficient and electrical resistivity were measured simultaneously using Ulvac ZEM-3 from room temperature to $1000 \mathrm{~K}$ under $\mathrm{He}$ atmosphere. The dimensions of the samples were near $10 \times 2.3 \times 2 \mathrm{~mm}$. High-density samples obtained after spark plasma sintering were cut into pellets with the diameter of $12.7 \mathrm{~mm}$ and thickness of $1.3 \mathrm{~mm}$. Their surfaces were polished before testing. The thermal conductivity was calculated using the formula $\kappa=D \times C_{p} \times d$. The density $(d)$ was measured utilizing the Archimedes method. The thermal diffusivity $(D)$ measurements were performed by a laser flash method using Netzsch LFA457 with Cape-Lehman and pulse correction, and all surfaces of the samples were coated with graphite to minimize emissivity. The high temperature specific heat $\left(C_{P}\right)$ was measured from room temperature to $1023 \mathrm{~K}$ by differential scanning calorimetry using Netzsch DSC 404F3 under the Ar flow rate of $5 \mathrm{~mL} \mathrm{~min}{ }^{-1}$. The mass for the high-temperature $C_{p}$ measurement was around $80 \mathrm{mg}$ and there was no mass lost for any of the samples after DSC measurements.

$X$-ray Diffraction (XRD) and TEM Anylysis: XRD patterns were collected from powders (before spark plasma sinstering) with $\mathrm{Cu} \mathrm{Ka}(\mathrm{I}=1.5418 \AA$ ) radiation, using Rigaku D/max $2250 \mathrm{~V}$ operating at $40 \mathrm{kV}$ and $20 \mathrm{~mA}$. No obvious orientations are observed in our polycrystalline samples. The ultrathin sectioning method has been used to prepare samples for TEM observations. The thin sectioned samples with a thickness of $35 \mathrm{~nm}$ have been obtained on a LEICA EM UC7 microtome by using a diamond knife at a cutting speed of $0.4 \mathrm{~mm} \mathrm{~s}^{-1}$. The sectioned sample was floated on the water and then transfered onto the Mo grid.

Low-Temperature Thermeoelctric Transport Properties: The thermal conductivity from 2 to $300 \mathrm{~K}$ was carried out using PPMS (Quantum Design) with the TTO option under high vacuum. Before the samples were measured, the system was corrected using a standard sample of metallic nickel. Sample dimensions were around $1.5 \mathrm{~mm} \times 1.5 \mathrm{~mm} \times$ $2.5 \mathrm{~mm}$. Heat capacities from 1.97 to $300 \mathrm{~K}$ were measured utilizing the Heat Capacity option on Quantum Design PPMS. Before testing samples, the baseline was scanned first. The mass for low-temperature measurements was around $11 \mathrm{mg}$. The carrier concentration $(p)$ and the hole mobility $\left(\mu_{H}\right)$ were calculated from $p=1 / e R_{H}$ and $m_{H}=s / p e$, where $\sigma$ is the electrical conductivity and $e$ is the elementary charge. The Hall resistance $\left(R_{H}\right)$ and the electrical conductivity were measured using the Hall and resistivity options for an $A C$ transport on Quantum Design PPMS, respectively. Sample dimensions for resistivity mensurements were $1.5 \mathrm{~mm} \times 1.5 \mathrm{~mm} \times 7.5 \mathrm{~mm}$ and for the Hall measurement were $1.5 \mathrm{~mm} \times 1 \mathrm{~mm} \times 6 \mathrm{~mm}$.

\section{Supporting Information}

Supporting Information is available from the Wiley Online Library or from the author.

\section{Acknowledgements}

This work was supported by National Basic Research Program of China (973-program) under Project No. 2013CB632501, and National Natural Science Foundation of China (NSFC) under Grant Nos. 51472262 and 51272270, the Key Research Program of Chinese Academy of Sciences (Grant No. KGZD-EW-T06). C.U. was supported by the Center for Solar and Thermal Energy Conversion, an Energy Frontier Research Center funded by the U.S. Department of Energy, Office of Basic Energy Sciences under Award No. DE_SC_0000957. G. J. S. was supported by EFRC Solid State Solar-Thermal Energy Conversion Center (S3TEC) under the Award No. DE-SC0001299. F.X. was supported by Shanghai technical platform for testing and characterization on inorganic materials under Grant No. 14DZ2292900.

Received: March 2, 2015

Revised: April 4, 2015

Published online: May 12, 2015

[1] L. E. Bell, Science 2008, 321, 1457.

[2] G. J. Snyder, E. S. Toberer, Nat. Mater. 2008, 7, 105.

[3] J. P. Heremans, M. S. Dresselhaus, L. E. Bell, D. T. Morelli, Nat. Nanotechnol. 2013, 8, 471 . 
[4] M. S. Dresselhaus, G. Chen, M. Y. Tang, R. G. Yang, H. Lee, D. Z. Wang, Z. F. Ren, J. P. Fleurial, P. Gogna, Adv. Mater. 2007, 19, 1043.

[5] J. P. Heremans, V. Jovovic, E. S. Toberer, A. Saramat, K. Kurosaki, A. Charoenphakdee, S. Yamanaka, G. J. Snyder, Science 2008, 321, 554.

[6] Y. Z. Pei, X. Shi, A , LaLonde, H. Wang, L. D. Chen, G. J. Snyder, Nature 2011, 473, 66.

[7] K. Biswas, J. He, I. D. Blum, C. I. Wu, T. P. Hogan, D. N. Seidman, V. P. Dravid, M. G. Kanatzidis, Nature 2012, 489, 414.

[8] L. D. Zhao, S. H. Lo, Y. Zhang, H. Sun, G. Tan, C. Uher, C. Wolverton, V. P. Dravid, M. G. Kanatzidis, Nature 2014, 508, 373.

[9] K. F. Hsu, S. Loo, F. Guo, W. Chen, J. S. Dyck, C. Uher, T. Hogan, E. K. Polychroniadis, M. G. Kanatzidis, Science 2004, 303, 818.

[10] A. I. Hochbaum, R. Chen, R. D. Delgado, W. Liang, E. C. Garnett, M. Najarian, A. Majumdar, P. Yang, Nature 2008, 451, 163.

[11] L. D. Hicks, M. S. Dresselhaus, Phys. Rev. B 1993, 47, 12727.

[12] J. Ma, O. Delaire, A. F. May, C. E. Carlton, M. A. McGuire, L. H. VanBebber, D. L. Abernathy, G. Ehlers, T. Hong, A. Huq, W. Tian, V. M. Keppens, H. Y. Shao, B. C. Sales, Nat. Nanotechol. 2013, 8, 445
[13] Y. He, T. Day, T. S. Zhang, H. L. Liu, X. Shi, L. D Chen, G. J. Snyder, Adv. Mater. 2014, 26, 3974.

[14] H. L. Liu, X. Shi, F. F. Xu, L. L. Zhang, W. Q. Zhang, L. D. Chen, Q. Li, C. Uher, T. Day, G. J. Snyder, Nat. Mater. 2012, 11, 422.

[15] C. G. Darwin, Philos. Mag. 1922, 43, 800.

[16] F. Zwicky, Phys. Rev. 1932, 40, 63.

[17] M. W. Geis, H. I. Smith, A. Argoitia, J. Angus, G. M. Ma, J. T. Glass, J. Bulter, C. J. Robinson, R. Pryor, Appl. Phys. Lett. 1991, 58, 2485.

[18] A. O. Er, J. Chen, J. Tang, P. M. Rentzepis, Appl. Phys. Lett. 2012, $100,151910$.

[19] E. Hirahara, J. Phys. Soc. Jpn. 1951, 6, 422.

[20] N. Vouroutzis, C. Manolikas, Phys. Status Solidi A 1989, 111, 491.

[21] J. L. Cohn, G. S. Nolas, V. Fessatidis, T. H. Metcalf, G. A. Slack, Phys. Rev. Lett. 1999, 82, 779.

[22] H. L. Liu, X. Yuan, P. Lu, X. Shi, F. Xu, Y. He, Y. Tang, S. Bai, W. Zhang, L. D. Chen, Y. Lin, L. Shi, H. Lin, X. Gao, X. Zhang, H. Chi, C. Uher, Adv. Mater. 2013, 25, 6607.

[23] D. R. Brown, T. Day, K. A. Borup, S. Christensen, B. B. Iversen, G. J. Snyder, APL Mater. 2013, 1, 052107.

[24] W. Koshibea, K. Tsutsui, S. Meakawa, Phys. Rev. B 2000, 62, 6869.

[25] G. A. Slack, in CRC Handbook of Thermoelectrics, (Ed: D. M. Rowe), CRC Press, Boca Raton, FL, USA 1995.

[26] L. Ratke, P. W. Voorhees, Growth and Coarsening: Ostwald Ripening in Material Processing, Springer, 2002. 